\title{
Alteration of Gut Microbiota and Efficacy of Probiotics in Functional Constipation
}

\author{
Chang Hwan Choi* and Sae Kyung Chang \\ Department of Internal Medicine, Chung-Ang University College of Medicine, Seoul, Korea
}

\author{
Article: Change of fecal flora and effectiveness of the short-term VSL\#3 probiotic treatment in patients with func- \\ tional constipation \\ Kim SE, Choi CS, Park KS, et al \\ (J Neurogastroenterol Motil 2015;21:111-120)
}

Functional constipation (FC) is a common symptom- based gastrointestinal (GI) disorder without organic abnormality, the prevalence of which is between $5 \%$ and $20 \%$ of the general population. ${ }^{1,2}$ The symptoms of constipation can be severe; thus, this disorder has a significant impact on patient's quality of life. ${ }^{3}$ The cause of FC remains unclear, especially for slow transit constipation. Alterations of the gut microbiota have been suggested as one possible pathophysiologic mechanism of FC. ${ }^{4,5}$ However, the quantitative and/or qualitative changes of the gut microbiota in $\mathrm{FC}$ are poorly understood, and many discrepancies exist in the literature.

In the current issue of the Journal of Neurogastroenterology and Motility, Kim et al ${ }^{6}$ have published a prospective study in which they investigated the characteristics of gut microbiota in patients with FC using quantitative real-time polymerase chain reaction. The authors found that Bifidobacterium and Bacteroides species were significantly less abundant in feces from patients with FC compared with healthy controls. However, no significant differences were observed regarding the proportion of Lactobacillus, Escherichia coli and Clostridium species. These results are partially consistent with previous studies that have analyzed the fecal microbiota in patients with $\mathrm{FC}^{7,8}$

Bifidobacterium and Lactobacillus have been shown to be significantly less abundant in adult patients with constipation. ${ }^{8}$ One study found that patients with constipation predominant-irritable bowel syndrome exhibited significantly less lactate-producing (bifidobacteria and lactobacilli) and lactate-utilizing bacteria in their feces compared with controls; moreover, the abundances of $\mathrm{H}_{2}$-consuming populations, methanogens and reductive acetogens were also significantly lower in these patients compared with controls. ${ }^{7}$ In contrast, a study of children with constipation found that the levels of bifidobacteria and clostridia were increased in

Received: November 27, 2014 Revised: December 13, 2014 Accepted: December 14, 2014

(c) This is an Open Access article distributed under the terms of the Creative Commons Attribution Non-Commercial License (http://creativecommons. org/licenses/by-nc/3.0) which permits unrestricted non-commercial use, distribution, and reproduction in any medium, provided the original work is properly cited.

*Correspondence: Chang Hwan Choi, MD, PhD

Department of Internal Medicine, Chung-Ang University College of Medicine, 102 Heukseok-ro, Dongjak-gu, Seoul 156-755, Korea

Tel: +82-2-6299-1418, Fax: +82-2-6299-2017, E-mail: gicch@cau.ac.kr

Financial support: None.

Conflicts of interest: None.

Author contributions: Chang Hwan Choi drafted and edited this article; and Sae Kyung Chang advised on editing draft.

ORCID: Chang Hwan Choi, http://orcid.org/0000-0001-7089-532X. 
their feces compared with healthy subjects. ${ }^{9}$ A cross-sectional pilot study using 16S rRNA gene pyrosequencing found that patients with constipation did not exhibit decreased levels of Lactobacillus and Bifidobacterium species, but did exhibit significantly decreased levels of Prevotella. ${ }^{10}$ Regarding methane, the presence of methanogenic bacteria has been shown to be higher in patients with slow transit constipation compared withnon-constipated controls. ${ }^{4}$ These alterations in gut microbiota might be either a cause of $\mathrm{FC}$ or a consequence of this condition. Differences in diet or delayed colon transit times might also lead to altered gut microbiota communities in patients with FC. A recent study using humanized (ex-germ-free mice colonized with human fecal microbiota) mice elegantly demonstrated that the composition of the gut microbiota is changed by altered GI motility. Specifically, humanized mice with faster transit due to the administration of polyethylene glycol or a nonfermentable cellulose-based diet exhibited similar changes in gut microbiota composition. ${ }^{11}$ Regardless of whether differences in gut microbiota are a cause or an effect of constipation, this body of evidence indicates that patients with FC exhibit different compositions of gut microbiota. However, the precise differences in composition are poorly characterized and conflicting data have been found. Thus, no definitive association between constipation and the abundance or lack of certain bacterial taxa in the gut microbiome has yet been established.

Kim et $\mathrm{al}^{6}$ investigated the efficacy of VSL\#3 probiotics on fecal microbiota and symptom improvement in patients with FC. Mean Bristol stool scores and the numbers of complete spontaneous bowel movements (CSBMs)/week increased significantly in patients with FC treated with VSL\#3. Relief of subjective symptoms including CSBM frequency, stool consistency, and abdominal bloating was reported in up to $70 \%$ of patients.

Gut microbiota are clearly associated with GI motility. ${ }^{12}$ In germ free rat model, intestinal bacteria promoted or suppressed the initiation and migration of the migrating myoelectric complex depending on the species. ${ }^{13}$ Gut microbiota can affect intestinal motor functions either directly or indirectly via mediators released by the gut immune response, the end-products of bacterial fermentation, or intestinal neuroendocrine factors. ${ }^{12,14-16}$

Some probiotic bacteria have been shown to stimulate intestinal motility in both animal and human studies. For instance, Escherichia coli Nissle 1917 supernatants enhanced colonic contractility by direct stimulation of smooth muscle cells. ${ }^{17}$ Intestinal bacteria have also been shown to stimulate myoelectric activity in the rat small intestine by promoting cyclic initiation and aboral propagation of migrating myoelectric complex. ${ }^{18}$ Moreover, Bifidobacterium animalis DN-173 010 was shown to shorten colonic transit time in healthy women, ${ }^{19}$ and Bifidobacterium lactis HN019 supplementation was shown to decrease whole gut transit time in adults with functional GI symptoms. ${ }^{20} \mathrm{~A}$ meta-analysis of 11 randomized controlled trials (RCTs), consisting of 464 subjects in total, concluded that probiotic supplementation decreased intestinal transit time. Compared with control subjects, patients with probiotic supplementation exhibited a standardized mean difference of 0.40 (95\% CI, 0.20-0.59; $P<0.001)$ in intestinal transit time; this reduction was even more significant in subjects with constipation and older subjects. ${ }^{21}$

Probiotics may accelerate intestinal transit by decreasing the proliferation of methanogens in patients with FC. Intestinal methanogens can slow intestinal transit via methane production, ${ }^{22}$ since overproduction of methane has been shown to directly inhibit motor activity. ${ }^{23}$ Consistent with these findings, the prevalence of methane-producing bacteria has been shown to be higher in patients with slow transit compared with healthy controls. ${ }^{4,24}$ Probiotics may also increase intestinal fermentation and there by enhance colonic peristalsis, which decreases transit time in FC. For instance, colonic fermentation of a physiologically relevant malabsorbed quantity of starch has been shown to significantly increase the number of high amplitude propagated contractions (HAPCs). ${ }^{25}$ Short chain fatty acids (SCFAs), a major end-product of bacterial fermentation, have been shown to increase parietal tone and stimulate ileal propulsive contractions when administered into the human terminal ileum. ${ }^{26,27}$ SCFAs have been proposed to stimulate motor responses by releasing neuroendocrine factors and/or lowering the intracolonic $\mathrm{pH} .^{27,28}$ Animal studies have demonstrated that SCFAs can initiate HAPCs and accelerate intestinal transit and motility via the intestinal release of 5-hydroxytryptamine. ${ }^{29}$ However, a recent human study found no significant differences compared with basal values regarding either the global motility index or the number of HAPCs after intracolonic infusion of acidic solutions or SCFAs. This finding indicates that the increased colonic motor activity induced by bacterial fermentation is not caused by acidification of the colonic contents or the production of SCFAs. ${ }^{30}$ Bacterial fermentation may influence intestinal motility through other mechanisms such as the production of gases, since colonic distension is associated with increased HAPCs, ${ }^{31}$ and/or other fermentation byproducts such as alcohols, ketones and aldehydes.

As aforementioned, intestinal transit can be accelerated by probiotics, however, some other probiotics have been shown to 
decrease bowel movements, and improve loose stool/diarrhea in patient with diarrhea predominant-irritable bowel syndrome. ${ }^{32,33}$ Thus, probiotics may have functions to normalize intestinal motility. Further studies are needed to evaluate the precise effects of gut microbiota and probiotics on GI motility, including the exact effects of fermentation in this process.

A few clinical trials have demonstrated beneficial effects of probiotics in patients with $\mathrm{FC}$. In a recent meta-analysis, $2 \mathrm{RCT}$ s demonstrated that probiotics enhanced the response to therapy in patients with FC. ${ }^{34-36}$ However, this result was not statistically significant (relative risk of failure to respond to therapy $=0.29$; 95\% CI, 0.07-1.12) and the data were quite heterogeneous. Two trials reported a significant increase in the mean number of bowel movements per week in total of 165 patients $(1.49$; 95\% CI, 1.02-1.96). ${ }^{34,36,37}$ Another meta-analysis of 14 RCTs (1,182 patients) reported that probiotics significantly reduced whole gut transit time by 12.4 hours, increased stool frequency by 1.3 bowel movements per week, and also improved stool consistency. ${ }^{38}$ However, these results also should be interpreted cautiously due to the high study heterogeneity and risk of bias. The efficacy of probiotics for treating FC has not yet been determined, and more RCTs are needed to evaluate the precise relationship between probiotics and FC.

Finally, Kim et $\mathrm{al}^{6}$ performed an elegant study of the compositional changes of the gut microbiota in FC, a process about which little is known, and demonstrated the effect of short-term treatment with VSL\#3 on FC using objective parameters such as CSBM and stool consistency, although this study did not show a generalized view of the diverse fecal microbiota and was not a placebo-controlled trial. These findings are cautiously promising with respect to the use of specific probiotic species in the management of FC. Additional well designed clinical studies are needed to determine the true efficacy of probiotics and to elucidate their mechanisms of action, which may include byproducts of bacterial metabolism such as gas and SCFA, in FC.

\section{References}

1. Lovell RM, Ford AC. Global prevalence of and risk factors for irritable bowel syndrome: a meta-analysis. Clin Gastroenterol Hepatol 2012;10:712-721, e4.

2. Suares NC, Ford AC. Prevalence of, and risk factors for, chronic idiopathic constipation in the community: systematic review and metaanalysis. Am J Gastroenterol 2011;106:1582-1591.

3. Belsey J, Greenfield S, Candy D, Geraint M. Systematic review: impact of constipation on quality of life in adults and children. Aliment Pharmacol Ther 2010;31:938-949.
4. Attaluri A, Jackson M, Valestin J, Rao SS. Methanogenic flora is associated with altered colonic transit but not stool characteristics in constipation without IBS. Am J Gastroenterol 2010;105:1407-1411.

5. Quigley EM. The enteric microbiota in the pathogenesis and management of constipation. Best Pract Res Clin Gastroenterol 2011;25: 119-126.

6. Kim SE, Choi SC, Park KS, et al. Change of fecal flora and effectiveness of the short-term VSL\#3 probiotic treatment in patients with functional constipation. J Neurogastroenterol Motil 2015;21:111120.

7. Chassard C, Dapoigny M, Scott KP, et al. Functional dysbiosis within the gut microbiota of patients with constipated-irritable bowel syndrome. Aliment Pharmacol Ther 2012;35:828-838.

8. Khalif IL, Quigley EM, Konovitch EA, Maximova ID. Alterations in the colonic flora and intestinal permeability and evidence of immune activation in chronic constipation. Dig Liver Dis 2005;37:838-849.

9. Zoppi G, Cinquetti M, Luciano A, Benini A, Muner A, Bertazzoni Minelli E. The intestinal ecosystem in chronic functional constipation. Acta Paediatr 1998;87:836-841.

10. Zhu L, Liu W, Alkhouri R, et al. Structural changes in the gut microbiome of constipated patients. Physiol Genomics 2014;46:679-686.

11. Kashyap PC, Marcobal A, Ursell LK, et al. Complex interactions among diet, gastrointestinal transit, and gut microbiota in humanized mice. Gastroenterology 2013;144:967-977.

12. Barbara G, Stanghellini V, Brandi G, et al. Interactions between commensal bacteria and gut sensorimotor function in health and disease. Am J Gastroenterol 2005;100:2560-2568.

13. Husebye E, Hellström PM, Sundler F, Chen J, Midtvedt T. Influence of microbial species on small intestinal myoelectric activity and transit in germ-free rats. Am J Physiol Gastrointest Liver Physiol 2001;280:G368-G380

14. Quigley EM. Microflora modulation of motility. J Neurogastroenterol Motil 2011;17:140-147.

15. Uribe A, Alam M, Johansson O, Midtvedt T, Theodorsson E. Microflora modulates endocrine cells in the gastrointestinal mucosa of the rat. Gastroenterology 1994;107:1259-1269.

16. Wu RY, Pasyk M, Wang B, et al. Spatiotemporal maps reveal regional differences in the effects on gut motility for Lactobacillus reuteri and rhamnosus strains. Neurogastroenterol Motil 2013;25:e205-e214.

17. Bär F, Von Koschitzky H, Roblick U, et al. Cell-free supernatants of Escherichia coli Nissle 1917 modulate human colonic motility: evidence from an in vitro organ bath study. Neurogastroenterol Motil 2009;21:559-566, e16-e17.

18. Husebye E, Hellström PM, Midtvedt T. Intestinal microflora stimulates myoelectric activity of rat small intestine by promoting cyclic initiation and aboral propagation of migrating myoelectric complex. Dig Dis Sci 1994;39:946-956.

19. Marteau $\mathrm{P}$, Cuillerier $\mathrm{E}$, Meance $\mathrm{S}$, et al. Bifidobacterium animalis strain DN-173 010 shortens the colonic transit time in healthy women: a double-blind, randomized, controlled study. Aliment Pharmacol Ther 2002;16:587-593.

20. Waller PA, Gopal PK, Leyer GJ, et al. Dose-response effect of Bifidobacterium lactis $\mathrm{HN} 019$ on whole gut transit time and functional gastrointestinal symptoms in adults. Scand J Gastroenterol 2011;46: 1057-1064

21. Miller LE, Ouwehand AC. Probiotic supplementation decreases in- 
testinal transit time: meta-analysis of randomized controlled trials. World J Gastroenterol 2013;19:4718-4725.

22. Sahakian AB, Jee SR, Pimentel M. Methane and the gastrointestinal tract. Dig Dis Sci 2010;55:2135-2143.

23. Pimentel M, Lin HC, Enayati $\mathrm{P}$, et al. Methane, a gas produced by enteric bacteria, slows intestinal transit and augments small intestinal contractile activity. Am J Physiol Gastrointest Liver Physiol 2006; 290:G1089-G1095.

24. Lee KM, Paik CN, Chung WC, Yang JM, Choi MG. Breath methane positivity is more common and higher in patients with objectively proven delayed transit constipation. Eur J Gastroenterol Hepatol 2013;25:726-732.

25. Jouët P, Sabaté JM, Coffin B, Lémann M, Jian R, Flourié B. Fermentation of starch stimulates propagated contractions in the human colon. Neurogastroenterol Motil 2011;23:450-456, e176.

26. Coffin B, Lémann M, Flourié B, Jouet P, Rambaud JC, Jian R. Local regulation of ileal tone in healthy humans. Am J Physiol 1997;272(1 Pt 1):G147-G153.

27. Kamath PS, Phillips SF, Zinsmeister AR. Short-chain fatty acids stimulate ileal motility in humans. Gastroenterology 1988;95:14961502.

28. Kruis W, Azpiroz F, Phillips SF. Contractile patterns and transit of fluid in canine terminal ileum. Am J Physiol 1985;249(2 Pt 1):G264G270.

29. Fukumoto S, Tatewaki M, Yamada T, et al. Short-chain fatty acids stimulate colonic transit via intraluminal 5-HT release in rats. Am J Physiol Regul Integr Comp Physiol 2003;284:R1269-R1276.

30. Jouet P, Moussata D, Duboc H, et al. Effect of short-chain fatty acids and acidification on the phasic and tonic motor activity of the human colon. Neurogastroenterol Motil 2013;25:943-949.
31. Bassotti G, Gaburri M, Imbimbo BP, Morelli A, Whitehead WE. Distension-stimulated propagated contractions in human colon. Dig Dis Sci 1994;39:1955-1960.

32. Cha BK, Jung SM, Choi CH, et al. The effect of a multispecies probiotic mixture on the symptoms and fecal microbiota in diarrheadominant irritable bowel syndrome: a randomized, double-blind, placebo-controlled trial. J Clin Gastroenterol 2012;46:220-227.

33. Whorwell PJ, Altringer L, Morel J, et al. Efficacy of an encapsulated probiotic Bifidobacterium infantis 35624 in women with irritable bowel syndrome. Am J Gastroenterol 2006;101:1581-1590.

34. Ford AC, Quigley EM, Lacy BE, et al. Efficacy of prebiotics, probiotics, and synbiotics in irritable bowel syndrome and chronic idiopathic constipation: systematic review and meta-analysis. Am J Gastroenterol 2014;109:1547-1561.

35. Koebnick C, Wagner I, Leitzmann P, Stern U, Zunft HJ. Probiotic beverage containing Lactobacillus casei Shirota improves gastrointestinal symptoms in patients with chronic constipation. Can J Gastroenterol 2003;17:655-659.

36. Sakai T, Makino H, Ishikawa E, Oishi K, Kushiro A. Fermented milk containing Lactobacillus casei strain Shirota reduces incidence of hard or lumpy stools in healthy population. Int J Food Sci Nutr 2011;62:423-430

37. Yang YX, He M, Hu G, et al. Effect of a fermented milk containing Bifidobacterium lactis DN-173010 on Chinese constipated women. World J Gastroenterol 2008;14:6237-6243.

38. Dimidi E, Christodoulides S, Fragkos KC, Scott SM, Whelan K. The effect of probiotics on functional constipation in adults: a systematic review and meta-analysis of randomized controlled trials. Am J Clin Nutr 2014;100:1075-1084. 